\title{
Article \\ Comparison of Antimicrobial Treatment Incidence Quantification Based on Detailed Field Data on Animal Level with the Standardized Methodology of the European Medicines Agency in Veal Calves, Switzerland, 2016-2018
}

\author{
Jens Becker * and Mireille Meylan (i) \\ Clinic for Ruminants, Vetsuisse-Faculty, University of Bern, Bremgartenstrasse 109a, 3012 Bern, Switzerland; \\ mireille.meylan@vetsuisse.unibe.ch \\ * Correspondence: jens.becker@vetsuisse.unibe.ch; Tel.: +41-31-631-23-42
}

check for

updates

Citation: Becker, J.; Meylan, M. Comparison of Antimicrobial Treatment Incidence Quantification Based on Detailed Field Data on Animal Level with the Standardized Methodology of the European Medicines Agency in Veal Calves, Switzerland, 2016-2018. Antibiotics 2021, 10, 832. https://doi.org/ 10.3390/antibiotics 10070832

Academic Editor: John Barlow

Received: 2 June 2021

Accepted: 5 July 2021

Published: 8 July 2021

Publisher's Note: MDPI stays neutral with regard to jurisdictional claims in published maps and institutional affiliations.

Copyright: (c) 2021 by the authors. Licensee MDPI, Basel, Switzerland. This article is an open access article distributed under the terms and conditions of the Creative Commons Attribution (CC BY) license (https:// creativecommons.org/licenses/by/ $4.0 /)$.
Abstract: Precise quantification of antimicrobial treatment incidence (TI) is crucial for benchmarking. Two widespread methods for treatment incidence quantification were compared for agreement. Field data were obtained from 38 veal farms from 2016 to 2018 (1905 calves, 1864 treatments). Calculation of $\mathrm{TI}_{\text {swiss }}$ for calves was based on detailed treatment records using pharmacokinetic values from the Swiss Veterinary Medicines Compendium. The method published by the European Medicines Agency was used to calculate $\mathrm{TI}$ in defined daily doses $\left(\mathrm{TI}_{\mathrm{DDD}}\right)$. For each calf and treatment, $\mathrm{TI}_{\text {swiss }}$ and $\mathrm{TI}_{\mathrm{DDD}}$ were calculated on level of the antimicrobial class, drug, application route, and farm. The quotient $(\mathrm{Q})$ of $\mathrm{TI}_{\text {swiss }}$ and $\mathrm{TI}_{\mathrm{DDD}}$ was calculated. Divergence in results between the two methods of $\leq 25 \%$ was arbitrarily set as good agreement. The agreement between $\mathrm{TI}_{\text {swiss }}$ and $\mathrm{TI}_{\mathrm{DDD}}$ was mostly good. On class level, good agreement was observed for treatments representing $71.5 \%$ of the $\mathrm{TI}_{\mathrm{DDD}}$, and $74.5 \%$ of the total $\mathrm{TI}_{\mathrm{DDD}}$ on drug level. Poor agreement was mainly observed for tylosin and sulfadimidine. The agreement was better for parenteral than for oral treatments $(81.6 \% \mathrm{vs} .72 .3 \%)$. For practically orientated calculation on farm level, good agreement was observed (77.5\% of the $\left.\mathrm{TI}_{\mathrm{DDD}}\right)$. The $\mathrm{TI}_{\mathrm{DDD}}$ method showed mostly good agreement, especially for parenteral treatments.

Keywords: treatment intensity; antimicrobial use; standardization; agreement

\section{Introduction}

Antimicrobial resistance (AMR) is likely to become an obstacle for human development, as antimicrobials may become ineffective for disease control and health care costs may rise considerably [1]. There is a large basis of evidence showing that antimicrobial use (AMU) is a major driver for the development of AMR in human and veterinary medicine through selection of resistant strains, which emphasizes the need to reduce AMU [2]. Different methods are used to quantify and compare AMU among countries, with the aim of developing strategies to monitor drug use and to identify excessive or inadequate use. Sales figures of antimicrobial therapeutic products have been published regularly for various countries for more than a decade [3-6]. However, an important limitation of the interpretation of sales figures is that antimicrobials cannot always be attributed to different animal species and production branches, and dose or duration of the treatments are rarely known. Thus, sales figures interpretation is difficult. Moreover, the amount of antimicrobials sold is not identical to the amount used, as, e.g., therapeutic products may expire before administration or packages may break. There is agreement that more detailed data than sales figures are needed to estimate AMU more precisely at the levels of the species, production branch, or farm [7].

Numerous methods and differing approaches have been developed to estimate AMU. However, comparison of the results of different methods often requires complicated conversion calculations or may be impossible. For example, the method for calculating the 
number of animal course doses is based on the summary of product characteristics (SPC, a legal document presenting the approved conditions of use and the properties of a drug [8]) and indicates the number of full-length treatments an animal was exposed to [9]. No direct comparison is possible with another method indicating the number of treatments per calves (NTPC) based on prescribed daily doses (PDD), even in the same country [10]. To use the NTPC method, very detailed data are necessary, including, e.g., the number of prescribed daily doses and an individual estimated weight for each treatment. Treatment incidence (TI) in animal daily doses (ADD) was given per calf-year in another study [11], and in the number of calves treated daily per 1000 calves at risk elsewhere [12]. Establishing a method which allows for comparison between studies and which requires limited data for calculation, which is easy to use and to interpret, and which applies to as many species and production branches as possible, is a major methodological challenge.

A method for TI quantification using defined daily doses $\left(\mathrm{TI}_{\mathrm{DDD}}\right)$ has been established by the European Medicines Agency [13,14]. It was developed based on the SPC from nine countries of the European Union [15], whereby a DDD is defined as the average estimated dose needed to treat one kilogram of the animal for the drug's main indication for one day. The subsequent calculation of TI from the amount of drug used includes a standard weight for the typical animal to be treated, the observation period (i.e., the number of days the calves were observed, and the treatments were recorded), and the number of calves present. The unit of the result is the number of treatment days per animal, or per animal-year if a factor for extension to a year's period is added to the formula. Values of DDD are provided by the EMA for most common drugs, for several species (cattle, swine, turkeys, and broilers), and administration routes (oral, parenteral; for cows additionally intramammary and intrauterine administration). This method allows for a direct result comparison among studies. However, there are limitations which may influence the results, e.g., the fact that different therapeutic products are used for a single drug in veterinary practice in Europe, and that their real potency, i.e., the actual dosages used as well as the weight of the animal at the time of treatment, may differ from the assumptions of the EMA based on average SPC values [11]. Therefore, the benefits of the method for comparing AMU are counterbalanced by reduced accuracy of AMU quantification. This may bias AMU reports and cause misinterpretation of the results. Nonetheless, for monitoring purposes, methods using DDD's have been adopted by the World Health Organization and by national monitoring programs of the Netherlands and Scandinavian countries $[2,4,5,16]$.

The aim of the present study was to investigate how precisely the calculated $\mathrm{TI}_{\mathrm{DDD}}$ reflects the $\mathrm{TI}_{\text {swiss }}$ calculated based on indications for use from the Swiss Veterinary Medicines Compendium [17] for one species and one production branch, using detailed field treatment data on individual animal level from veal calves in Switzerland.

\section{Material and Methods}

\subsection{Data Collection and Treatment Recording}

Antimicrobial treatments (hereafter 'treatments') were recorded on 38 Swiss veal calf fattening farms between October 2016 and July 2018 in the frame of a previous study [18]. Briefly, farms were either intervention farms implementing a novel concept for veal calf fattening or control farms, and all calves were fattened according to the label IP-SUISSE which requires higher standards for animal welfare and sustainability than the Swiss legislation [19]. All components and procedures of the concept were approved by the competent authority (authorization number BE 71/16). Only the essential information for the present study is presented here. The novel concept was developed to mitigate the effects of the risk factors for increased AMU and mortality identified in previous studies [11,20]. The 'outdoor veal calf' concept is described in detail elsewhere [18]. The main hypothesis for that study was that AMU would be lower by at least $50 \%$ on intervention farms compared to controls. Antimicrobial use was assessed in detail during farm visits, which were performed at higher frequency than in other veal calf studies [12,21,22]. Each farm was followed for a minimum of twelve consecutive months and visited once monthly by one 
member of the study team (consisting of 3 veterinarians). A total of 535 farm visits were conducted. Treatments were recorded in detail by use of a specially customized booklet on each farm in accordance with statutory requirements (identification number of the treated animal(s), first and last administration date, name of therapeutic product, dosage, indication for treatment, administration route (oral vs. parenteral (i.e., intravenous, intramuscular or subcutaneous); and withdrawal period). Additionally, information on administration type (individual or group treatment), observed signs of disease, and treatment outcome were recorded. Only treatments with therapeutic products containing antimicrobials were recorded. Treatment modalities were determined at the farm veterinarians' discretion, and the record booklets were filled in by the veterinarians with the farmers. The study team neither provided advice nor commented on the treatments. Dosages of the drugs were determined by farm veterinarians through estimation of the live body weight of the calves at the time of treatment. Diagnostic investigations such as culture of nasopharyngeal swabs or fluids obtained by bronchoalveolar lavage, and determination of susceptibility to antimicrobial drugs was at the discretion of the treating veterinarians; susceptibility testing was mostly not performed prior to treatment. A total of 1905 calves were enrolled, of which 731 received antimicrobial treatment (38.4\%); a total of 1864 treatments were recorded. Treatment records were revised at each visit and incomplete records were completed with the farmer or the respective veterinarian, if necessary.

\subsection{Treatment Incidence Quantification}

The treatment incidences $\left(\mathrm{TI}_{\mathrm{Swiss}}\right.$ and $\left.\mathrm{TI}_{\mathrm{DDD}}\right)$ were calculated separately for each calf and each treatment. Treatments with therapeutic products containing two drugs were handled as two separate treatments in both methods. The unit of TI is the number of daily doses per calf (for $\mathrm{TI}_{\text {swiss }}$ and $\mathrm{TI}_{\mathrm{DDD}}$ ) $[13,14]$.

\subsection{TI Quantification Based on Data from the Swiss Veterinary Medicines Compendium $\left(T_{\text {swiss }}\right)$}

All used drugs were registered with the Swiss authority for licensing therapeutic products (Swissmedic) [23]. Information on the pharmacokinetics of each product were available online (Swiss Veterinary Medicines Compendium, https:/ / www.vetpharm.uzh. ch/tak/clinidoc.htm, accessed on 2 May 2020) [17]), and treatment according to the recommended dose for the weight of the animal as estimated by the treating veterinarian was assumed. The maintenance period of a drug was defined as the period (in days) during which the minimal concentration required for successful treatment was obtained. Drugs were administered either once or several times with varying intervals between applications. Oral and parenteral route were used for application. Therefore, the following categories of administration modalities were differentiated:

(A) Daily oral or parenteral drug administration: for drugs which were administered orally or parenterally once daily during several consecutive days, the maintenance period was equivalent to the number of days the drug was administered.

(B) Repeated parenteral administration at two-day intervals: for drugs which were administered parenterally at two-day intervals (i.e., drugs with a maintenance period of two days), each administration day was counted as two treatment days.

(C) Single administration of long-acting preparations: for drugs which were administered once, the maintenance period indicated in the Swiss Veterinary Medicines Compendium was used. If this maintenance period was indicated to be a range of days (for example 5-7 days), the average maintenance period was used (i.e., 6 days).

(D) Repeated administration of drugs with overlapping maintenance periods: for drugs which were prescribed to be administered at a certain interval but of which the maintenance period exceeds this interval, $\mathrm{TI}_{\text {swiss }}$ was obtained as follows: number of applications * interval between applications + (maintenance period-interval between applications). Thus, for a drug with a maintenance period of 2.5 days which was administered twice at an interval of two days, the $\mathrm{TI}_{\text {swiss }}$ is $4.5[2 \times 2+(2.5-2)=4.5]$. 
(E) Drugs without indication of a maintenance period: for tulathromycin, no maintenance period is indicated in the Swiss Veterinary Medicines Compendium. It was arbitrarily set to be 10 days after single parenteral administration of $2.5 \mathrm{mg} / \mathrm{kg}$ based on the following observations: after single application of $2.5 \mathrm{mg} / \mathrm{kg}$ plasma concentrations were $>20 \mathrm{ng} / \mathrm{mL}$ for up to 12 days $[24,25]$. Pulmonary concentrations (in pulmonary epithelial lining fluid) were estimated to be approximatively 100 times higher (i.e., $2 \mu \mathrm{g} / \mathrm{mL}$ ) [26]. The minimal inhibitory concentrations were $1-4 \mu \mathrm{g} / \mathrm{mL}$ for Pasteurellaceae [27]. Thus, pulmonary concentrations met the average minimum inhibitory concentration for approximatively 10 days.

\subsection{Treatment Incidence Quantification with the EMA Method ( $T I_{D D D}$ )}

The same dataset (as for the calculation of $\mathrm{TI}_{\text {swiss }}$ ) was used. Values for DDD were available for all drugs used and their respective administration routes. For calculation, the following Formula (1) was used:

$$
\mathrm{TI}_{\mathrm{DDD}}=\frac{\text { total amount of drug administered }(\mathrm{mg})}{D D D\left(\frac{m g}{k g}\right) * \text { observation period (days) } * \text { number of calves present } * \text { standard weight }(\mathrm{kg})}
$$

In contrast to the calculation of $\mathrm{TI}_{\text {swiss, }}$ the standard weight at treatment of $80 \mathrm{~kg}$ defined by the EMA was applied here for all calves and all treatments [13,14].

\subsection{Statistical Analyses}

For every treatment, a quotient $(\mathrm{Q})$ of $\mathrm{TI}_{\mathrm{swiss}}$ and $\mathrm{TI}_{\mathrm{DDD}}\left(\mathrm{Q}=\mathrm{TI}_{\mathrm{swiss}} / \mathrm{TI} \mathrm{I}_{\mathrm{DDD}}\right)$ was calculated. When $\mathrm{Q}=1, \mathrm{TI}_{\text {swiss }}$ equals $\mathrm{TI}_{\mathrm{DDD}}$; when $\mathrm{Q}<1$, the estimation of $\mathrm{TI}_{\text {by }} \mathrm{TI}_{\text {swiss }}$ is lower than the one by $\mathrm{TI}_{\mathrm{DDD}}$; and when $\mathrm{Q}>1$, the estimation of $\mathrm{TI}_{\text {by }} \mathrm{TI}_{\mathrm{swiss}}$ is higher than the one by $\mathrm{TI}_{\mathrm{DDD}}$. Treatments were grouped by antimicrobial class, drug, application route, and farm ID. Subsequently, the number of treatments, the sum of $\mathrm{TI}_{\mathrm{swiss}}$, the sum of $\mathrm{TI}_{\mathrm{DDD}}$, and the percentage of the total TI per group were calculated. For each group, the median of $Q$ and the interquartile range (IQR) of $Q$ were calculated. For antimicrobial classes, drugs, application routes or farms, respectively, where $Q$ values indicated a discrepancy between $\mathrm{TI}_{\text {swiss }}$ and $\mathrm{TI}_{\mathrm{DDD}} \leq 0.25(25 \%)$, the methods were arbitrarily considered to be in good agreement with each other. In observer reliability statistics, a discrepancy up to 0.20 is still considered 'almost perfect' agreement, and a discrepancy up to 0.40 as 'substantial' agreement [28-30]. Drugs used less than 10 times were excluded from further analyses, regardless of the TI. Additionally, EMA DDD values were directly compared with 'daily doses' calculated based on the extensive dataset of $\mathrm{TI}_{\text {swiss, }}$ as described above. For this calculation of 'daily doses', the equation for $\mathrm{TI}_{\mathrm{DDD}}$ calculation was rearranged by solving for the position of the factor 'DDD'. This factor was renamed 'daily dose', and values were obtained by inserting the required values 'amount of drug used', 'observation period', and 'number of calves present'. The factor 'standard weight' was replaced by the estimated mean live body weight at treatment (separately for oral and parenteral treatments; $111.0 \mathrm{~kg}$ for oral and $124.1 \mathrm{~kg}$ for parenteral treatments). These standardized weights were calculated based on an assumed start weight at the beginning of the fattening period of $72.1 \mathrm{~kg}$, a constant weight gain throughout the fattening period, and the average treatment time point as described elsewhere (mean weight and age at treatment \pm standard deviation $119.3 \mathrm{~kg} \pm 43.4 ; 72.1$ days \pm 29.1 ) [18]. Calculations were performed using Microsoft Excel ${ }^{\circledR}$ (Microsoft, Redmont, WA, USA), and 'R' Version 3.5.1 (R Core Team 2020, $R$ Foundation for Statistical Computing, Vienna, Austria). 


\section{Results}

The most frequently used antimicrobial classes by $\mathrm{TI}_{\mathrm{DDD}}$ were tetracyclines $(45.6 \%)$, penicillins (19.1\%), and macrolides (12.4\%). The most frequently used antimicrobial classes by $\mathrm{TI}_{\text {swiss }}$ were tetracyclines $(32.5 \%)$, macrolides $(25.5 \%)$ and sulfonamides $(20.2 \%)$.

Antimicrobial treatments representing $73.3 \%$ of the $\mathrm{TI}_{\mathrm{DDD}}$ were administered orally through the feed (mixed with milk or milk replacer), whereas $26.7 \%$ were administered parenterally through either intravenous, intramuscular or subcutaneous injection. Antimicrobial treatments representing $71.5 \%$ of the $\mathrm{TI}_{\mathrm{DDD}}$ were administered as group treatments, and $28.5 \%$ as individual treatments. Group treatments were considerably more frequently administered orally than parenterally ( $95.9 \%$ vs. $4.1 \%$ of the $\left.\mathrm{TI}_{\mathrm{DDD}}\right)$. Individual treatments were mainly administered parenterally ( $83.4 \%$ vs. $16.6 \%$ of the $\left.\mathrm{TI}_{\mathrm{DDD}}\right)$.

Grouped by antimicrobial class, treatments corresponding to $71.5 \%$ (all treatments) differed by $\leq 25 \%$ between $\mathrm{TI}_{\text {swiss }}$ and $\mathrm{TI}_{\mathrm{DDD}}$ (Table 1 ).

For the comparison of $\mathrm{TI}_{\text {swiss }}$ and $\mathrm{TI}_{\mathrm{DDD}}$ on drug level, treatments with the following drugs were excluded due to low numbers of observations (number of observations): neomycin, spectinomycin, lincomycin, enrofloxacin, sulfamethoxypyridazine (1); procaine benzylpenicillin/benzathin, cefquinome, sulfadoxine (2); amoxicillin/clavulanic acid, sulfadiazine, sulfadoxine (3); ceftiofour (7). Of the remaining 1837 treatments, the most frequently used drugs by $\mathrm{TI}_{\mathrm{DDD}}$ were chlortetracycline $(28.4 \%)$, amoxicillin $(15.4 \%)$, and oxytetracycline $(8.7 \%)$. The most frequently used drugs by $\mathrm{TI}_{\text {swiss }}$ were chlortetracycline $(21.5 \%)$, sulfadimidine $(17.1 \%)$, and tylosin $(14.1 \%)$. Grouped by drug, treatments corresponding to $74.5 \%$ of the total $\mathrm{TI}_{\mathrm{DDD}}$ differed by $\leq 25 \%$ (Table 2 ).

The most frequently drugs used for oral application were chlortetracycline $(38.4 \%$ of the $\left.\mathrm{TI}_{\mathrm{DDD}}\right)$, doxycycline $(11.6 \%)$, and sulfadimidine $(11.3 \%)$, and treatments corresponding to $72.3 \%$ of the total $\mathrm{TI}_{\mathrm{DDD}}$ differed by $\leq 25 \%$ (Table 3 ).

The most frequently drugs used for parenteral application were oxytetracycline $(33.8 \%$ of the $\left.\mathrm{TI}_{\mathrm{DDD}}\right)$, tulathromycin $(19.5 \%)$, and procaine benzylpenicillin $(14.2 \%)$. Of those, treatments corresponding to $81.6 \%$ of the total $\mathrm{TI}_{\mathrm{DDD}}$ differed by $\leq 25 \%$ (Table 4 ).

Of 38 participating farms, the three farms with the highest $\mathrm{TI}$ by $\mathrm{TI}_{\mathrm{DDD}}$ accounted for $13.3 \%, 12.3 \%$, and $11.4 \%$ of the total $\mathrm{TI}_{\mathrm{DDD}}$. Two of those were part of the three farms with the highest $\mathrm{TI}$ by $\mathrm{TI}_{\text {swiss }}$, which accounted for $15.0 \%, 12.9 \%$, and $11.4 \%$ of the total $\mathrm{TI}_{\text {swiss }}$. Grouped by farm ID, treatments corresponding to $75.5 \%$ of the total $\mathrm{TI}_{\mathrm{DDD}}$ values differed by $\leq 25 \%$ (Table 5 ).

Table 6 presents the EMA DDD values as well as the hypothetical 'daily doses' for $\mathrm{TI}_{\text {swiss. }}$ According to the previous results, the newly calculated 'daily doses' are closely comparable for most drugs, except for sulfonamides, most macrolides and streptomycin. There is overall better agreement for parenteral DDD's with 'daily doses' values than for oral values. 


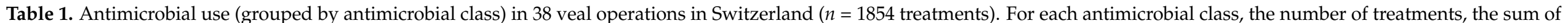

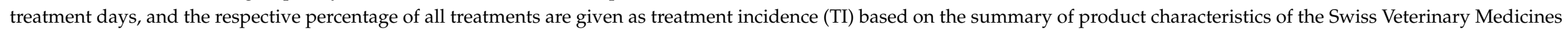

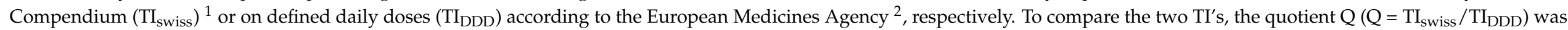

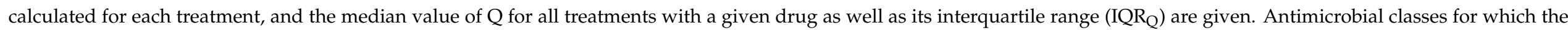
agreement between $\mathrm{TI}_{\text {swiss }}$ and $\mathrm{TI}_{\mathrm{DDD}}$ was good (maximal discrepancy $\leq 25 \%$ ) are highlighted in grey.

\begin{tabular}{|c|c|c|c|c|c|c|c|c|}
\hline $\begin{array}{c}\text { Agreement between } \mathrm{TI}_{\text {swiss }} \text { and } \\
\mathrm{TI}_{\mathrm{DDD}} \text { Methods }\end{array}$ & Antimicrobial Class & $\mathbf{n}^{3}$ & $\begin{array}{l}\text { Sum of } \mathrm{TI}_{\text {swiss }} \\
\text { (Days) }\end{array}$ & $\%$ of Total $\mathrm{TI}_{\text {swiss }}{ }^{4}$ & $\begin{array}{c}\text { Sum of } \\
\text { TI DDD (Days) }_{\text {DD }}\end{array}$ & $\%$ of Total TI $\mathrm{DDD}^{5}$ & $\begin{array}{l}\text { Median } \\
\text { of } Q\end{array}$ & IQR of $Q$ \\
\hline \multirow{2}{*}{ Discrepancy $>25 \%\left(\mathrm{TI}_{\mathrm{DDD}}<\mathrm{TI}_{\text {swiss }}\right)$} & Macrolides & 372 & 3157 & 25.5 & 1548.91 & 12.4 & 1.94 & $1.56-7.42$ \\
\hline & Sulfonamides & 343 & 2499 & 20.2 & 1351.75 & 10.8 & 1.87 & $1.42-2.33$ \\
\hline \multirow{5}{*}{$\begin{array}{l}\mathrm{TI}_{\text {swiss }} \text { in good agreement with } \mathrm{TI}_{\mathrm{DDD}} \\
\quad \text { (maximal discrepancy } \leq 25 \% \text { ) }\end{array}$} & Phenicols & 10 & 43.26 & 0.4 & 36.71 & 0.3 & 1.16 & $1.16-1.16$ \\
\hline & $\begin{array}{c}\text { Amino- } \\
\text { glycosides }\end{array}$ & 32 & 150 & 1.2 & 140.87 & 1.1 & 1.14 & $0.80-2.07$ \\
\hline & Florfenicols & 101 & 330.93 & 2.7 & 439.64 & 3.5 & 1.07 & $0.57-1.16$ \\
\hline & Tetracyclines & 593 & 4017 & 32.5 & 5682.93 & 45.6 & 0.80 & $0.63-0.92$ \\
\hline & Penicillins & 292 & 1638 & 13.3 & 2383.37 & 19.1 & 0.76 & $0.49-0.95$ \\
\hline \multirow{2}{*}{ Discrepancy $>25 \%\left(\mathrm{TI}_{\mathrm{DDD}}>\mathrm{TI}_{\mathrm{swiss}}\right)$} & $\begin{array}{c}\text { Fluoro- } \\
\text { quinolones }\end{array}$ & 51 & 143 & 1.2 & 238.53 & 1.9 & 0.72 & $0.48-1.74$ \\
\hline & $\begin{array}{c}\text { Diamino- } \\
\text { pyrimidins }\end{array}$ & 60 & 379 & 3.1 & 636.43 & 5.1 & 0.60 & $0.58-0.60$ \\
\hline Sum $^{6}$ & & 1854 & $12,357.19$ & 100.1 & $12,459.14$ & 99.8 & & \\
\hline Sum & & 1079 & 6322.19 & 51.3 & 8922.05 & 71.5 & & \\
\hline
\end{tabular}

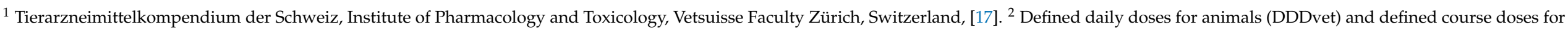

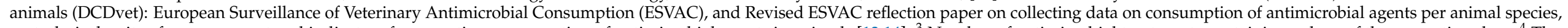

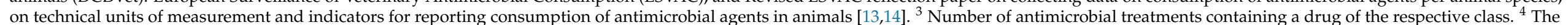

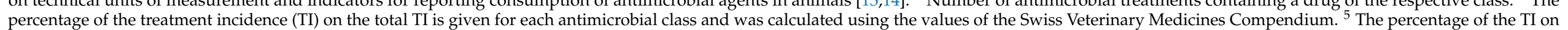

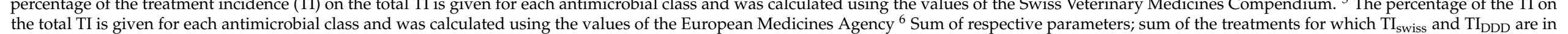
good agreement (highlighted in grey) 


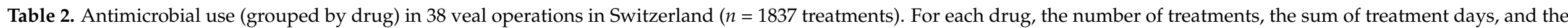

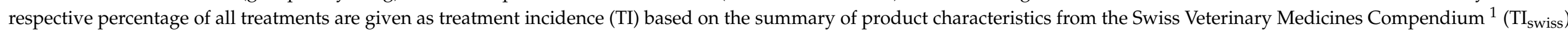

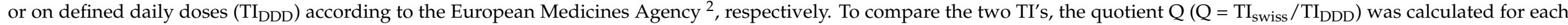

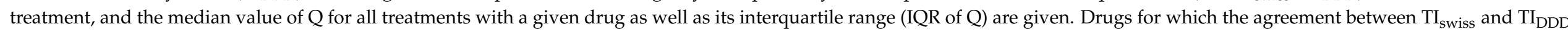
was good (maximal discrepancy $\leq 25 \%$ ) are highlighted in grey.

\begin{tabular}{|c|c|c|c|c|c|c|c|c|c|}
\hline $\begin{array}{l}\text { Agreement between } \mathrm{TI}_{\text {swiss }} \text { and } \\
\text { TI }_{\text {DDD Methods }}\end{array}$ & Drug & Antimicrobial Class & $\mathrm{n}^{3}$ & $\underset{\text { (Days) }}{\text { Sum of } \mathrm{TI}_{\text {swiss }}}$ & $\stackrel{\%}{\%} \underset{\text { of Total } \mathrm{TI}_{\text {swiss }} 4}{4}$ & $\begin{array}{l}\text { Sum of } \\
\text { TIDDD } \\
\text { (Days) }\end{array}$ & $\%$ of Total TI $\mathrm{DDD}^{5}$ & $\begin{array}{c}\text { Median } \\
\text { of } Q\end{array}$ & IQR of $Q$ \\
\hline \multirow{2}{*}{$\begin{array}{l}\text { Discrepancy }>25 \% \\
\left(\mathrm{TI}_{\mathrm{DDD}}<\mathrm{TI}_{\text {swiss }}\right)\end{array}$} & Tylosin & Macrolides & 222 & 1735 & 14.1 & 368.64 & 3.0 & 7.39 & $5.47-8.04$ \\
\hline & Sulfadimidine & Sulfonamides & 278 & 2109 & 17.1 & 1037.98 & 8.4 & 1.89 & $1.50-2.69$ \\
\hline \multirow{10}{*}{$\begin{array}{c}\mathrm{TI}_{\mathrm{swiss}} \text { in good agreement } \\
\text { with } \mathrm{TI}_{\mathrm{DDD}} \\
\text { (maximal discrepancy } \leq 25 \% \text { ) }\end{array}$} & Phthalylsulfathiazole & Sulfonamides & 56 & 370 & 3.0 & 301.49 & 2.4 & 1.25 & $1.20-1.25$ \\
\hline & Tilmicosin & Macrolides & 13 & 39 & 0.3 & 37.14 & 0.3 & 1.20 & $0.96-1.20$ \\
\hline & Tulathromycin & Macrolides & 46 & 466 & 3.8 & 626.97 & 5.0 & 1.20 & $0.78-1.20$ \\
\hline & $\begin{array}{c}\text { Dehydro- } \\
\text { streptomycin }\end{array}$ & Aminoglycosides & 30 & 145 & 1.2 & 138.82 & 1.1 & 1.07 & $0.80-1.90$ \\
\hline & Florfenicol & Florfenicols & 101 & 330.93 & 2.7 & 439.64 & 3.5 & 1.07 & $0.57-1.16$ \\
\hline & Marbofloxacin & Fluoroquinolones & 31 & 80 & 0.6 & 97.33 & 0.8 & 0.90 & $0.72-1.80$ \\
\hline & $\begin{array}{c}\text { Oxy- } \\
\text { tetracycline }\end{array}$ & Tetracyclines & 207 & 813 & 6.6 & 1086.86 & 8.7 & 0.87 & $0.52-1.14$ \\
\hline & Amoxicillin & Penicillins & 223 & 1389.5 & 11.3 & 1910.6 & 15.4 & 0.82 & $0.64-0.95$ \\
\hline & Doxycycline & Tetracyclines & 77 & 558 & 4.5 & 1069.52 & 8.6 & 0.80 & $0.80-0.80$ \\
\hline & $\begin{array}{c}\text { Chlor- } \\
\text { tetracycline }\end{array}$ & Tetracyclines & 309 & 2646 & 21.5 & 3526.55 & 28.4 & 0.78 & $0.63-0.92$ \\
\hline \multirow{2}{*}{$\begin{array}{l}\text { Discrepancy }>25 \% \\
\left(\mathrm{TI}_{\text {DDD }}>\mathrm{TI}_{\text {swiss }}\right)\end{array}$} & $\begin{array}{c}\text { Procaine } \\
\text { benzylpenicillin }\end{array}$ & Penicillins & 64 & 232 & 1.9 & 456.08 & 3.7 & 0.49 & $0.35-0.75$ \\
\hline & Danofloxacin & Fluoroquinolones & 19 & 61 & 0.5 & 138.22 & 1.1 & 0.47 & $0.34-0.63$ \\
\hline Sum $^{6}$ & & & 1837 & 12313.69 & 100 & 12425.14 & 100 & & \\
\hline Sum & & & 1103 & 6880.69 & 55.9 & 9271.63 & 74.5 & & \\
\hline
\end{tabular}

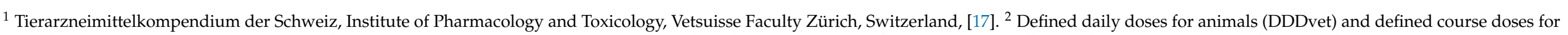

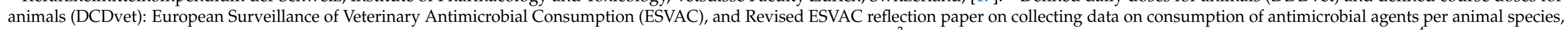

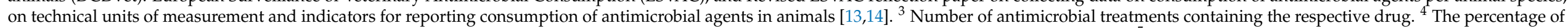

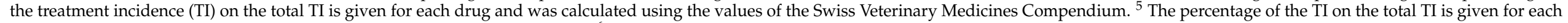

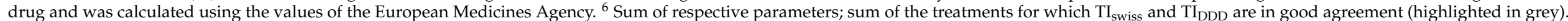




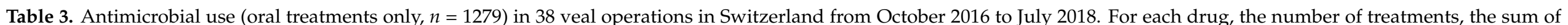

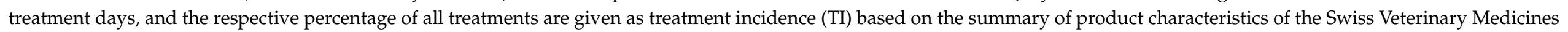

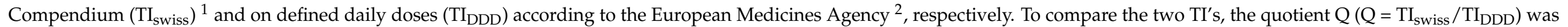

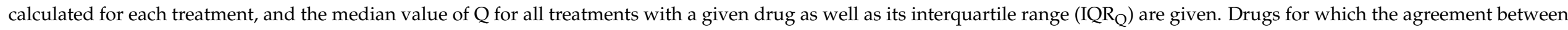
$\mathrm{TI}_{\text {swiss }}$ and $\mathrm{TI}_{\mathrm{DDD}}$ was good (maximal discrepancy $\leq 25 \%$ ) are highlighted in grey.

\begin{tabular}{|c|c|c|c|c|c|c|c|c|c|}
\hline $\begin{array}{c}\text { Agreement between } \mathrm{TI}_{\text {swiss }} \text { and } \\
\text { TI }_{\text {DDD Methods }}\end{array}$ & Drug & $\begin{array}{l}\text { Antimircrobial } \\
\text { Class }\end{array}$ & $n^{3}$ & $\begin{array}{c}\text { Sum of } \\
\text { TI }_{\text {swiss }} \text { (Days) }\end{array}$ & $\begin{array}{c}\% \\
\text { of Total TI } \\
\text { swiss }\end{array}$ & $\begin{array}{c}\text { Sum of } \\
\text { TI DDD }_{\text {Days) }}\end{array}$ & $\stackrel{\%}{\text { of Total TI DDD }}{ }^{5}$ & $\begin{array}{l}\text { Median } \\
\text { of } Q\end{array}$ & IQR of $Q$ \\
\hline \multirow{3}{*}{ Discrepancy $>25 \%\left(\mathrm{TI}_{\mathrm{DDD}}<\mathrm{TI}_{\text {swiss }}\right)$} & Tylosin & Macrolides & 218 & 1729 & 17.4 & 361.72 & 3.9 & 7.39 & $5.47-8.04$ \\
\hline & Spiramycine & Macrolides & 91 & 917 & 9.2 & 516.16 & 5.6 & 1.94 & $1.94-1.94$ \\
\hline & Sulfadimidine & Sulfonamides & 276 & 2105 & 21.1 & 1035.8 & 11.3 & 1.89 & $1.50-2.69$ \\
\hline \multirow{3}{*}{$\begin{array}{l}\mathrm{TI}_{\text {swiss }} \text { in good agreement with } \mathrm{TI}_{\mathrm{DDD}} \\
\quad \text { (maximal discrepancy } \leq 25 \%)\end{array}$} & $\begin{array}{c}\text { Phthalyl- } \\
\text { sulfathiazole }\end{array}$ & Sulfonamides & 56 & 370 & 3.7 & 301.49 & 3.3 & 1.25 & $1.20-1.25$ \\
\hline & Amoxicillin & Penicillins & 197 & 1267.5 & 12.7 & 1749.05 & 19 & 0.82 & $0.64-0.95$ \\
\hline & $\begin{array}{c}\text { Chlor- } \\
\text { tetracycline }\end{array}$ & Tetracyclines & 309 & 2646 & 26.6 & 3526.55 & 38.4 & 0.78 & $0.63-0.92$ \\
\hline Discrepancy $>25 \%\left(\mathrm{TI}_{\mathrm{DDD}}>\mathrm{TI}_{\mathrm{swiss}}\right)$ & Trimethoprim & Diaminopyrimidins & 55 & 368 & 3.7 & 622.67 & 6.8 & 0.60 & $0.58-0.60$ \\
\hline Sum ${ }^{6}$ & & & 1279 & 9960.5 & 100 & 9182.96 & 99.9 & & \\
\hline Sum & & & 639 & 4841.5 & 48.6 & 6646.61 & 72.3 & & \\
\hline
\end{tabular}

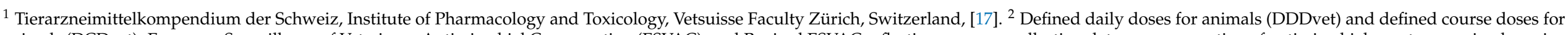

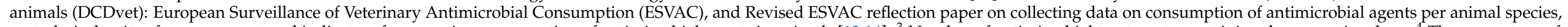

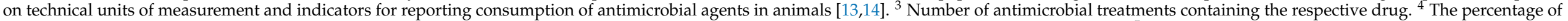

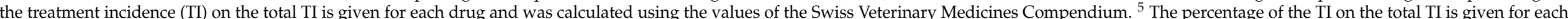

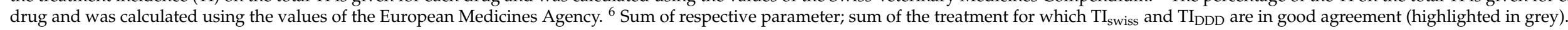




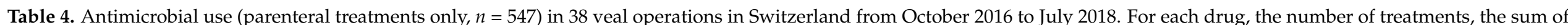

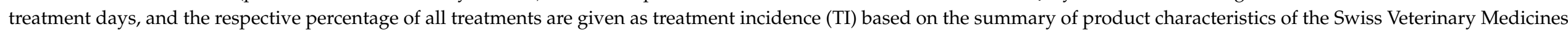

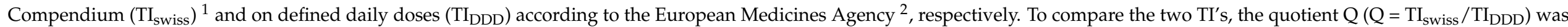

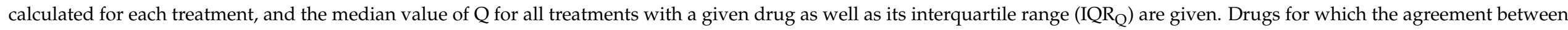
$\mathrm{TI}_{\text {swiss }}$ and $\mathrm{TI}_{\mathrm{DDD}}$ was good (maximal discrepancy $\leq 25 \%$ ) are highlighted in grey.

\begin{tabular}{|c|c|c|c|c|c|c|c|c|c|}
\hline $\begin{array}{c}\text { Agreement between } \mathrm{TI}_{\text {swiss }} \text { and } \mathrm{TI}_{\mathrm{DDD}} \\
\text { Methods }\end{array}$ & Drug & $\underset{\text { Class }}{\text { Antimircrobial }}$ & $n^{3}$ & $\begin{array}{l}\text { Sum of } \\
\text { TI }_{\text {swiss }} \\
\text { (Days) }\end{array}$ & of Total $\operatorname{TI}_{\text {swiss }} 4$ & $\begin{array}{l}\text { Sum of } \\
\text { TI DDD } \\
\text { (Days) }\end{array}$ & $\begin{array}{c}\% \\
\text { of Total } \\
\text { TI }_{\text {DDD }} 5\end{array}$ & $\begin{array}{l}\text { Median } \\
\text { of } Q\end{array}$ & $\begin{array}{l}\text { IQR } \\
\text { of } Q\end{array}$ \\
\hline \multirow{6}{*}{$\begin{array}{c}\mathrm{TI}_{\text {swiss }} \text { in good agreement with } \\
\mathrm{TI}_{\mathrm{DDD}}(\text { maximal discrepancy } \leq 25 \%)\end{array}$} & Tilmicosin & Macrolides & 13 & 39 & 1.7 & 37.14 & 1.2 & 1.20 & $0.96-1.20$ \\
\hline & $\begin{array}{l}\text { Tulathro- } \\
\text { mycin }\end{array}$ & Macrolides & 46 & 466 & 20 & 626.97 & 19.5 & 1.20 & $0.78-1.20$ \\
\hline & Florfenicol & Phenicols & 10 & 43.26 & 1.9 & 36.71 & 1.1 & 1.16 & $1.16-1.16$ \\
\hline & $\begin{array}{c}\text { Dehydro- } \\
\text { streptomcin }\end{array}$ & Aminoglycosides & 30 & 145 & 6.2 & 138.82 & 4.3 & 1.07 & $0.80-1.90$ \\
\hline & Florfenicol & Florfenicols & 101 & 330.93 & 14.2 & 439.64 & 13.7 & 1.07 & $0.57-1.16$ \\
\hline & $\begin{array}{l}\text { Marbo- } \\
\text { floxacin }\end{array}$ & Fluoroquinolones & 31 & 80 & 3.4 & 97.33 & 3 & 0.90 & $0.72-1.80$ \\
\hline \multirow{4}{*}{ Discrepancy $>25 \%\left(\mathrm{TI}_{\mathrm{DDD}}>\mathrm{TI}_{\text {swiss }}\right)$} & Amoxicillin & Penicillins & 26 & 122 & 5.2 & 161.55 & 5 & 0.89 & $0.68-0.89$ \\
\hline & $\begin{array}{l}\text { Oxy- } \\
\text { tetracycline }\end{array}$ & Tetracyclines & 207 & 813 & 34.9 & 1086.86 & 33.8 & 0.87 & $0.52-1.14$ \\
\hline & $\begin{array}{c}\text { Procaine } \\
\text { benzylpenicillin }\end{array}$ & Penicillins & 64 & 232 & 9.9 & 456.08 & 14.2 & 0.49 & $0.35-0.76$ \\
\hline & Danofloxacin & Fluoroquinolones & 19 & 61 & 2.6 & 138.22 & 4.3 & 0.47 & $0.34-0.63$ \\
\hline Sum & & & 464 & 2039.19 & 87.5 & 2625.02 & 81.6 & & \\
\hline
\end{tabular}

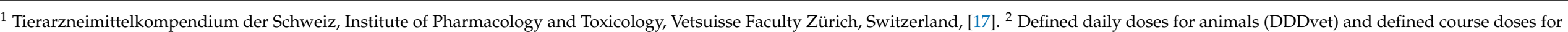

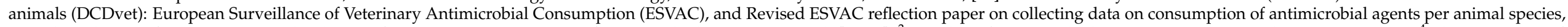

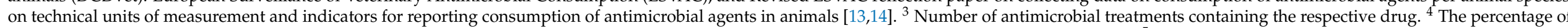

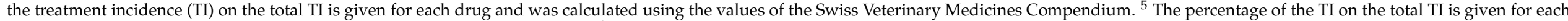

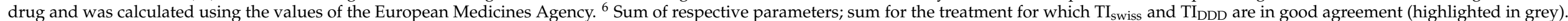




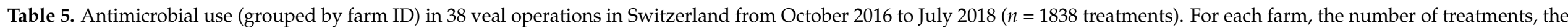

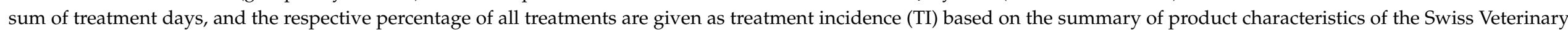

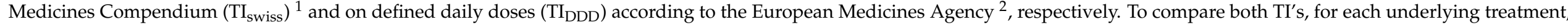

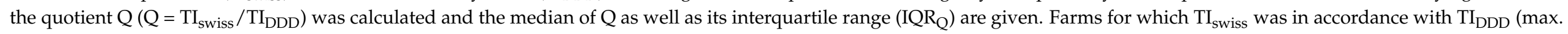
under/overestimation $\leq 25 \%$ ) are highlighted in grey.

\begin{tabular}{|c|c|c|c|c|c|c|c|c|}
\hline $\begin{array}{c}\text { Agreement between } \mathrm{TI}_{\text {swiss }} \text { and } \\
\text { TI }_{\text {DDD Methods }}\end{array}$ & Farm ID & $n^{3}$ & $\begin{array}{c}\text { Sum } \\
\text { of TI } \\
\text { (Days) }\end{array}$ & $\begin{array}{c}\% \\
\text { of Total TI } \\
\text { swiss }\end{array}$ & $\begin{array}{c}\text { Sum } \\
\text { of TI DDD (Days) }\end{array}$ & $\begin{array}{c}\% \\
\text { of Total } \\
\text { TI }_{\text {DDD }}\end{array}$ & Median of $Q$ & IQR of $Q$ \\
\hline \multirow{4}{*}{$\begin{array}{l}\text { Discrepancy }>25 \% \\
\left(\mathrm{TI}_{\mathrm{DDD}}<\mathrm{TI}_{\mathrm{swiss}}\right)\end{array}$} & 23 & 44 & 292.75 & 2.4 & 103.09 & 0.8 & 7.00 & $2.35-27.33$ \\
\hline & 24 & 19 & 93 & 0.8 & 49.55 & 0.4 & 2.00 & $1.73-2.67$ \\
\hline & 34 & 22 & 102.15 & 0.8 & 77.9 & 0.6 & 1.78 & $0.80-2.17$ \\
\hline & 3 & 46 & 256.41 & 2.1 & 272.98 & 2.2 & 1.30 & $0.92-1.80$ \\
\hline \multirow{17}{*}{$\begin{array}{l}\mathrm{TI}_{\text {swiss }} \text { in good agreement with } \\
\text { TI } \\
\text { (maximal discrepancy } \leq 25 \% \text { ) }\end{array}$} & 21 & 221 & 1575 & 12.9 & 1630.22 & 13.3 & 1.20 & $0.80-1.25$ \\
\hline & 18 & 17 & 41 & 0.3 & 33.28 & 0.3 & 1.17 & $0.65-3.50$ \\
\hline & 27 & 152 & 1400 & 11.4 & 1180.39 & 9.6 & 1.16 & $0.92-1.94$ \\
\hline & 25 & 230 & 1835.21 & 15.0 & 1397.21 & 11.4 & 1.14 & $0.89-2.69$ \\
\hline & 32 & 41 & 259 & 2.1 & 253.25 & 2.1 & 1.14 & 0.95-1.14 \\
\hline & 33 & 19 & 44.5 & 0.4 & 46.62 & 0.4 & 1.08 & $0.87-1.08$ \\
\hline & 36 & 130 & 858 & 7.0 & 709.91 & 5.8 & 1.05 & $0.80-2.06$ \\
\hline & 37 & 64 & 431 & 3.5 & 352.17 & 2.9 & 0.96 & $0.85-2.06$ \\
\hline & 38 & 165 & 711.56 & 5.8 & 825.21 & 6.7 & 0.90 & $0.63-1.89$ \\
\hline & 6 & 22 & 73.76 & 0.6 & 91.95 & 0.8 & 0.87 & $0.57-1.11$ \\
\hline & 17 & 11 & 34 & 0.3 & 33.31 & 0.3 & 0.87 & $0.56-16.21$ \\
\hline & 28 & 19 & 108.5 & 0.9 & 145.31 & 1.2 & 0.87 & $0.64-0.87$ \\
\hline & 31 & 70 & 494 & 4.0 & 558.5 & 4.6 & 0.87 & $0.61-1.30$ \\
\hline & 22 & 23 & 220 & 1.8 & 293.51 & 2.4 & 0.82 & $0.71-0.82$ \\
\hline & 26 & 44 & 161.5 & 1.3 & 226.45 & 1.8 & 0.81 & $0.54-1.30$ \\
\hline & 29 & 176 & 1380.63 & 11.3 & 1508.42 & 12.3 & 0.80 & $0.64-1.90$ \\
\hline & 16 & 16 & 68 & 0.6 & 94.89 & 0.8 & 0.77 & $0.63-0.98$ \\
\hline
\end{tabular}


Table 5. Cont.

\begin{tabular}{|c|c|c|c|c|c|c|c|c|}
\hline $\begin{array}{l}\text { Agreement between } \mathrm{TI}_{\text {swiss }} \text { and } \\
\mathrm{TI}_{\text {DDD Methods }}\end{array}$ & Farm ID & $\mathrm{n}^{3}$ & $\begin{array}{c}\text { Sum } \\
\text { of TI } \\
\text { (Days) }\end{array}$ & $\begin{array}{c}\% \\
\text { of Total TI } \\
\text { swiss }\end{array}$ & $\begin{array}{l}\text { Sum } \\
\text { of TIDDD (Days) }\end{array}$ & $\begin{array}{c}\% \\
\text { of Total } \\
\text { TI }_{\text {DDD }} 5\end{array}$ & Median of Q & IQR of $Q$ \\
\hline \multirow{6}{*}{ Overestimation of TI by $>25 \%$} & 20 & 14 & 59.89 & 0.5 & 99.02 & 0.8 & 0.66 & $0.46-0.94$ \\
\hline & 39 & 67 & 521.82 & 4.3 & 602.72 & 4.9 & 0.63 & $0.47-1.40$ \\
\hline & 5 & 24 & 143.63 & 1.2 & 378.22 & 3.1 & 0.52 & $0.32-0.80$ \\
\hline & 14 & 15 & 42.5 & 0.3 & 92.68 & 0.8 & 0.42 & $0.35-0.52$ \\
\hline & 15 & 13 & 53.5 & 0.4 & 137.16 & 1.1 & 0.38 & $0.38-0.38$ \\
\hline & 40 & 23 & 66.25 & 0.5 & 124.22 & 1.0 & 0.35 & $0.35-0.89$ \\
\hline Sum ${ }^{6}$ & & 1838 & $12,243.69$ & 100 & $12,256.84$ & 100 & & \\
\hline Sum & & 1439 & 9809.16 & 80.1 & 9474.36 & 77.5 & & \\
\hline
\end{tabular}

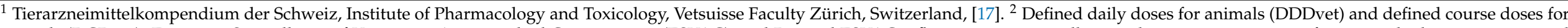

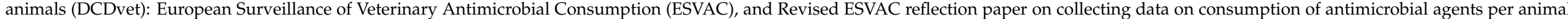

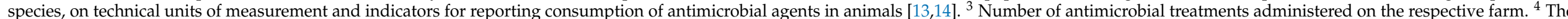
(TI) on the tol TI is given for each farm and was calcula

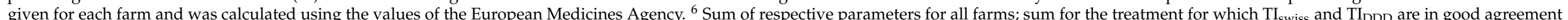
(highlighted in grey) 
Table 6. Antimicrobial drugs for oral and parenteral use and their mean daily dose calculated from detailed field treatment data of 38 Swiss veal operations ( $n=1854$ treatments) and from the summary of product characteristics of the Swiss Veterinary Medicines Compendium ${ }^{1}$. These hypothetical 'Swiss' daily doses were obtained by solving the European Medicines Agency's equation for treatment incidence ${ }^{2}$ calculation for the position of the factor of defined daily doses (DDD) and integrating all remaining variables from field treatment data.

\begin{tabular}{|c|c|c|c|c|c|}
\hline $\begin{array}{c}\text { Administration } \\
\text { Route }\end{array}$ & Drug & Antimicrobial Class & $n^{3}$ & Mean Daily Dose & DDD \\
\hline \multirow{9}{*}{ Oral Use } & Tylosin & Macrolides & 218 & 6.09 & 41 \\
\hline & Sulfadimidin & Sulfonamides & 221 & 34.17 & 105 \\
\hline & Spiramycine & Macrolides & 91 & 14.21 & 35 \\
\hline & Sulfadimidin * & Sulfonamides & 55 & 14.6 & 30 \\
\hline & Phthalylsulfathiazole * & Sulfonamides & 56 & 14.74 & 25 \\
\hline & Chlortetracycline & Tetracyclines & 309 & 21.35 & 22 \\
\hline & Amoxicillin & Penicillins & 197 & 20.72 & 20 \\
\hline & Trimethoprim * & Diaminopyrimidins & 55 & 5.84 & 4.8 \\
\hline & Doxycycline & Tetracyclines & 77 & 12.92 & 10 \\
\hline \multirow{8}{*}{ Parenteral Use } & Tilmicosin & Macrolides & 13 & 2.45 & 4 \\
\hline & Dehydrostreptomycin & Aminoglycosides & 30 & 15.5 & 25 \\
\hline & Marbofloxacin & Fluoroquinolones & 31 & 2.66 & 3.6 \\
\hline & Tulathromycin & Macrolides & 46 & 0.25 & 0.3 \\
\hline & Amoxicillin & Penicillins & 26 & 7.17 & 8.3 \\
\hline & Oxytetracycline & Tetracyclines & 207 & 5.72 & 6.5 \\
\hline & Florfenicol & Florfenicols & 101 & 11.68 & 13 \\
\hline & Danofloxacin & Fluoroquinolones & 19 & 2.59 & 1.9 \\
\hline
\end{tabular}

${ }^{1}$ Tierarzneimittelkompendium der Schweiz, Institute of Pharmacology and Toxicology, Vetsuisse Faculty Zürich, Switzerland, [17].

2 Defined daily doses for animals (DDDvet) and defined course doses for animals (DCDvet): European Surveillance of Veterinary Antimicrobial Consumption (ESVAC), and Revised ESVAC reflection paper on collecting data on consumption of antimicrobial agents per animal species, on technical units of measurement and indicators for reporting consumption of antimicrobial agents in animals $[13,14]$.

${ }^{3}$ Number of antimicrobial treatments containing the respective drug. ${ }^{*}$ In combination with trimethoprim or a sulfonamide, respectively.

\section{Discussion}

Overall, a good agreement between the two methods for TI calculation was observed. It is improbable to achieve a full agreement $(100 \%)$ between the two methods for TI quantification as both are based on a number of assumptions. The $\mathrm{TI}_{\mathrm{DDD}}$ method was developed to allow for standardization of TI with a multitude of products licensed in different countries. When using the treatment record dataset of the present study, the method provided good standardization. Limited deviations to the 'true' TI may be acceptable, as the achieved standardization outweighs the negative effects of deviations. Calculating and reporting standardized TI is crucial for benchmarking, and allows for comparison of TI between production branches, farms, and countries as well as over time. This way, it is possible, e.g., to classify farmers as high, intermediate, or low users of antimicrobials in the frame of monitoring and prevention programs.

Discrepancy between TI values was quantified similarly to another study by calculation of the quotient $\mathrm{Q}$ [31]. We considered a divergence of up to $25 \%$ between $\mathrm{TI}_{\text {swiss }}$ and $\mathrm{TI}_{\mathrm{DDD}}$ to represent a good agreement. This threshold was arbitrarily set yet follows the principles of classical correlation statistics. The threshold may be a subject of discussion. When considering the range of countries and the number of therapeutic products for which the $\mathrm{TI}_{\mathrm{DDD}}$ method was developed, we suggest that this threshold is suitable, and the $\mathrm{TI}_{\mathrm{DDD}}$ method can be considered to serve its purpose. If the threshold was extended to $\leq 30 \%$, the percentage of treatments for which both methods provide agreeing results would increase to a small extent only (by 1.9\% due to treatments of the class of fluoroquinolones and $5.8 \%$ due to one farm, respectively). In contrast, if the threshold value was set to $\leq 20 \%$, the agreement between the two methods would be moderately to considerably lower. This would 
almost exclusively be due to a lower agreement for chlortetracycline ( $-28.4 \%$ and $-38.4 \%$ when grouped by class and for oral treatments, respectively), although the agreement for the antimicrobial class 'tetracyclines' as a whole would not be affected and the agreement for oxytetracycline would still be classified as 'good'. The lower agreement would also be due to reclassification of treatments with the class 'penicillins' $(-19.1 \%)$. The agreement for amoxicillin would still be classified as 'good', but not for procaine benzylpenicillin. Tetracylines were frequently used in the calves enrolled in this study. Therefore, a shift from 'agreement' to 'non-agreement' of one drug belonging to the tetracycline class may have a great impact if analyzed on drug level, and no or little impact if analyzed on the level of the antimicrobial class and for parenteral treatments only. Using the most practical approach where agreement was calculated on farm level, i.e., on a set of treatments that had been used in real life, a lower threshold of $\leq 20 \%$ would entail a minimal change $(0.8 \%$ of the total $\mathrm{TI}_{\mathrm{DDD}}$ would no longer be classified as 'good agreement'). This shows that both methods provide similar results especially when used on class level and for the purpose of TI calculation of farm level treatment records. Alternatively, TI calculation may also be conducted with prescribed amounts of antimicrobials, implying the risk of those not reflecting accurately the truly administered amounts [32].

All methods that are used for TI calculation are based on assumptions to varying degrees. This includes the 'real' TI where values of the Swiss Veterinary Medicines Compendium are used for calculation. Correspondingly, none of the methods should be regarded as gold standard and be used as reference. By agreeing on one method for TI quantification in the field, the same assumptions would be made in all calculations. This would increase comparability between countries and production types.

In many veal calf studies, farm visits are conducted at intervals of several months [22]. We conducted labor-intensive monthly farm visits aimed principally at following up closely on the treatment history of the farms. By revising each of close to 2000 treatment records one month or less after administration and cross-checking animal ear tag numbers, we achieved good data quality on calf level. Clarifications could be addressed with the farmer or the farm veterinarian within short delays, if necessary. This way, we were able to eliminate as many inaccuracies as possible before losing track on treatment modalities as may be the case when collecting data retrospectively. In contrast to a similar study in swine [33], treatments were recorded prospectively to avoid loss of information due to incomplete data storage.

For correct $\mathrm{TI}_{\mathrm{DDD}}$ calculation, the observation period is needed $[13,14]$. By using data from the animal traffic database and slaughter data, we obtained the exact observation period for each calf. Therefore, we are confident that the data quality was good and an accurate estimation of $\mathrm{TI}_{\mathrm{DDD}}$ was obtained which qualified for comparison.

The results of this study are restricted to the production branch of veal calves. It would be useful to perform similar studies using detailed treatment record datasets from populations of adult cattle, or other species the EMA provides DDD values for. According to the species or production branch, different DDD values and standard weights at treatment apply. Therefore, it is not possible to estimate the agreement of the methods for those species based on the present results.

The repertoire of drugs used in the present study as well as their distribution are in accordance with the results of another recent study conducted in a different subset of Swiss veal farms [34]. This underlines that our dataset represents commonly applied drugs. In the present study, a few drugs were excluded to avoid drawing conclusions on an entire antimicrobial class from a low number of observed treatments. In regard to highest priority critically important antimicrobials (HPCIA), the use of third and fourth generation cephalosporins was very low and such treatments were excluded due to the low numbers of observations. Therefore, the two methods of TI quantification cannot be compared for this antimicrobial class. Agreement between $\mathrm{TI}_{\mathrm{Swiss}}$ and $\mathrm{TI}_{\mathrm{DDD}}$ for fluoroquinolones as a group was below the limit for good agreement with a median $Q$ value of 0.72 , with large differences between the two drugs analyzed (median $Q=0.90$ for marbofloxacin and 0.47 
for danofloxacin). The guidelines of the IP-SUISSE label applied in all farms of the study require that macrolides, quinolones and third and fourth generation cephalosporins may only be used in exceptional cases following a written justification by the farm veterinarian. This may have contributed to a limited use of cephalosporins; however, their use was also low in another study where mostly non-IP-SUISSE farms were followed, suggesting that there are sufficient alternatives to replace cephalosporins in practice [21]. Furthermore, the use of fluoroquinolones, which are used for individual treatments as cephalosporins higher generation, was distinctly higher with $6-7 \%$ (depending on the quantification method) of parenteral treatments. Likewise, the use of macrolides was high for oral and for parenteral treatments, and thus label restrictions obviously did not halt the use of all HPCIA in study farms.

In addition to the methodological comparison of $\mathrm{TI}_{\mathrm{swiss}}$ and $\mathrm{TI}_{\mathrm{DDD}}$, we calculated 'daily doses' based on the treatment data of the present study to illustrate the agreement of the methods on that level (Table 6). We explicitly did not aim at suggesting alternative defined daily doses, we took advantage of the fact that, in contrast to other field studies, it was possible to calculate 'daily doses' from calf-level treatment data because the exact observation period was available This direct comparison did not reveal a trend in any direction (consistently higher or lower TI values with one method), which underlines the specificities of each drug and thus the fact that each drug must be considered separately.

Besides a general good agreement, a pronounced discrepancy was observed for few antimicrobial drugs and classes. Mainly, the discrepancy between $\mathrm{TI}_{\text {swiss }}$ and $\mathrm{TI}_{\mathrm{DDD}}$ for tylosin and sulfonamides negatively influenced the overall agreement of the two methods. Therapeutic products containing tylosin alongside other antimicrobials are licensed with lower daily doses in Switzerland compared with EMA values, as a synergistic effect of the different antimicrobials to certain bacteria had been suggested by some (despite a lack of evidence to support this statement) [13,31,32]. Therefore, lower oral daily doses for tylosin are indicated in the Swiss Veterinary Medicines Compendium than in the EMA method. This may explain why the discrepancy in tylosin TI's between the two methods exceeds $25 \%$ by far. For sulfonamides, many different products are available. The EMA provides separate DDD values for sulfonamides and trimethoprim when used alone and in combination. To improve the agreement for sulfonamides, specific DDD values would have to be assigned to each licensed therapeutic product currently on sale. This approach would lead to a more precise estimation of $\mathrm{TI}_{\mathrm{DDD}}$. However, the large number of licensed commercial therapeutic products would make the determination of DDD values for each product excessively labor- and cost-intensive.

Especially for the most practical approach where treatments were grouped on farm level, $77.5 \%$ of the total $\mathrm{TI}_{\mathrm{DDD}}$ values represented treatments which diverged by less than $25 \%$ from $\mathrm{TI}_{\text {swiss }}$ values. This further supports that the standardized $\mathrm{TI}_{\mathrm{DDD}}$ method is useful for TI estimation. Frequently, therapeutic products used for metaphylactic treatment contain two or more drugs (for example SK 60 ad. us. vet ${ }^{\circledR}$, Biokema SA, 1023 Crissier, CH) containing spiramycine and chlortetracycline), leading to simultaneous administration of the two drugs. For this product, median $Q$ values were above and below 1.00 (1.94 and 0.78 for spiramycin and chlortetracycline, respectively); thus, this is one example where the two components of a product equal out each other to a certain extent. This is, however, not applicable to all products containing more than one drug.

Despite the well-known limitations of the EMA method [13,14], the availability of one single standardized method providing results that can be compared within and among countries and production branches in the course of years is of utmost importance. Especially for the veal calf production branch which was observed to use relatively high amounts of antimicrobials, accurate quantification methods are needed to document the evolution of AMU over time. 


\section{Conclusions}

In summary, reporting $\mathrm{AMU}$ by using the $\mathrm{TI}_{\mathrm{DDD}}$ method is reasonably accurate for most investigated drugs in veal calf production branch based on our dataset. Trading off the information needed to calculate $\mathrm{TI}_{\mathrm{DDD}}$ with the output it generates, this method provides an efficient way to obtain reliable and comparable results.

Author Contributions: The authors contributed as follows: conceptualization of the study (J.B. and M.M.), analyses and writing of the manuscript (J.B.), and proof-reading (M.M.). All authors have read and agreed to the published version of the manuscript.

Funding: This work was supported by the Migros Genossenschaftsbund and IP-SUISSE as well as the Federal Office of Agriculture, and the National Research Program 'Antimicrobial Resistance' (NRP 72) of the Swiss National Science Foundation (407240_167083).

Data Availability Statement: The data presented in this study are available on request.

Acknowledgments: We thank Meike Mevissen for advice regarding pharmacokinetics and all farmers for support with data collection.

Conflicts of Interest: The authors declare no conflict of interest.

\section{References}

1. Cassini, A.; Högberg, L.D.; Plachouras, D.; Quattrocchi, A.; Hoxha, A.; Simonsen, G.S.; Colomb-Cotinat, M.; Kretzschmar, M.E.; Devleesschauwer, B.; Cecchini, M.; et al. Attributable Deaths and Disability-Adjusted Life-Years Caused by Infections with Antibiotic-Resistant Bacteria in the EU and the European Economic Area in 2015: A Population-Level Modelling Analysis. Lancet Infect. Dis. 2019, 19, 56-66. Available online: https:/ /linkinghub.elsevier.com/retrieve/pii/S1473309918306054 (accessed on 27 September 2020). [CrossRef]

2. WHO. WHO Report on Surveillance of Antibiotic Consumption; WHO: Geneva, Switzerland, 2018; p. 128. Available online: https:/ / apps.who.int/iris/bitstream/handle/10665/277359/9789241514880-eng.pdf (accessed on 2 May 2020).

3. Bundesamt für Lebensmittelsicherheit und Veterinärwesen ARCH-Vet. ARCH-Vet; 2020; pp. 1-19. Available online: https: //www.blv.admin.ch/blv/de/home/tiere/tierarzneimittel/antibiotika/vertrieb.html (accessed on 1 September 2020).

4. MARAN Monitoring of Antimicrobial Resistance and Antibiotic Asage in Animals in The Netherlands in 2017. 2018; p. 79. Available online: https://www.wur.nl/upload_mm/7/b/0/5e568649-c674-420e-a2ca-acc8ca56f016_Maran\%202018.pdf (accessed on 27 September 2020).

5. Henius, A.E.; Pedersen, K.; Jensen, L.B. Danmap 2017. 2017. Available online: https://backend.orbit.dtu.dk/ws/files/16171365 6/Rapport_DANMAP_2017.pdf (accessed on 14 April 2021).

6. European Medicines Agency Sales of Veterinary Antimicrobial Agents in 31 European Countries in 2017: Trends from 2010-2017; Ninth ESVAC Rep. EMA/294674/2019; 2019; p. 106. Available online: https://www.ema.europa.eu/en/documents/report/salesveterinary-antimicrobial-agents-31-european-countries-2017_en.pdf (accessed on 27 September 2020).

7. Stebler, R.; Carmo, L.P.; Heim, D.; Naegeli, H.; Eichler, K.; Muentener, C.R. Extrapolating Antibiotic Sales to Number of Treated Animals: Treatments in Pigs and Calves in Switzerland, 2011-2015. Front. Vet. Sci. 2019, 6, 318. Available online: https: / / www.frontiersin.org/article/10.3389/fvets.2019.00318/full (accessed on 2 May 2020). [CrossRef]

8. EMA Summary of Product Characteristics. 2020. Available online: https://www.ema.europa.eu/en/glossary/summary-productcharacteristics (accessed on 30 December 2020).

9. ANSES Suivi des Ventes de Médicaments Vétérinaires Contenant des Antibiotiques en France en 2015. [Tracking Sales of Veterinary Medicines Containing Antibiotics in France in 2015]. 2016. Available online: https://www.anses.fr/fr/content/suivides-ventes-dantibiotiques-veterinaires (accessed on 2 May 2020).

10. Jarrige, N.; Cazeau, G.; Morignat, E.; Chanteperdrix, M.; Gay, E. Quantitative and qualitative analysis of antimicrobial usage in white veal calves in France. Prev. Vet. Med. 2017, 144, 158-166. [CrossRef] [PubMed]

11. Lava, M.; Schüpbach-Regula, G.; Steiner, A.; Meylan, M. Antimicrobial drug use and risk factors associated with treatment incidence and mortality in Swiss veal calves reared under improved welfare conditions. Prev. Vet. Med. 2016, 126, 121-130. [CrossRef] [PubMed]

12. Pardon, B.; Catry, B.; Dewulf, J.; Persoons, D.; Hostens, M.; De Bleecker, K.; Deprez, P. Prospective study on quantitative and qualitative antimicrobial and anti-inflammatory drug use in white veal calves. J. Antimicrob. Chemother. 2012, 67, 1027-1038. [CrossRef] [PubMed]

13. EMA Defined Daily Doses for Animals (DDDvet) and Defined Course Doses for Animals (DCDvet): European Surveillance of Veterinary Antimicrobial Consumption (ESVAC). 2016; Volume 44, pp. 13-18. Available online: http:/ / www.ema.europa.eu/ docs/en_GB/document_library/Other/2016/04/WC500205410.pdf (accessed on 2 May 2020). 
14. EMA Revised ESVAC Reflection Paper on Collecting Data on Consumption of Antimicrobial Agents per Animal Species, on Technical Units of Measurement and Indicators for Reporting Consumption of Antimicrobial Agents in Animals. EMA/286416/2012-Rev.1; 2013; Volume 44, pp. 1-29. Available online: https:/ /www.ema.europa.eu/en/documents / scientificguideline/revised-european-surveillance-veterinary-antimicrobial-consumption-esvac-reflection-paper-collecting_en.pdf (accessed on 28 June 2019).

15. EMA Principles on Assignment of Defined Daily Dose for Animals (DDDvet) and Defined Course Dose for Animals (DCDvet). Ema/710019/2014; 2015; Volume 44, p. 68. Available online: http://www.ema.europa.eu/docs/en_GB/document_library/ Scientific_guideline/2015/06/WC500188890.pdf (accessed on 11 May 2020).

16. NORM/NORM-VET Usage of Antimicrobial Agents and Occurrence of Antimicrobial Resistance in Norway. Tromsø/Oslo. 2017. Available online: https:/ /www.vetinst.no/en/surveillance-programmes/norm-norm-vet-report (accessed on 10 April 2021).

17. Institute of Pharmacology and Toxicology Vetsuisse Faculty Switzerland Tierarzneimittelkompendium der Schweiz. 2020. Available online: www.tierarzneimittel.ch (accessed on 28 August 2020).

18. Becker, J.; Schüpbach-Regula, G.; Steiner, A.; Perreten, V.; Wüthrich, D.; Hausherr, A.; Meylan, M. Effects of the novel concept 'outdoor veal calf' on antimicrobial use, mortality and weight gain in Switzerland. Prev. Vet. Med. 2020, 176, 104907. [CrossRef]

19. IP-SUISSE Richtlinien. IP-SUISSE Richtlinien Tierhaltung Rindvieh; Schweine; Schafe; Geflügel; 2019; pp. 13-15. Available online: https:/ / www.ipsuisse.ch/richtlinien-tierhaltung/ (accessed on 20 June 2019).

20. Lava, M.; Pardon, B.; Schüpbach-Regula, G.; Keckeis, K.; Deprez, P.; Steiner, A.; Meylan, M. Effect of calf purchase and other herd-level risk factors on mortality, unwanted early slaughter, and use of antimicrobial group treatments in Swiss veal calf operations. Prev. Vet. Med. 2016, 126, 81-88. [CrossRef] [PubMed]

21. Schnyder, P.; Schönecker, L.; Schüpbach-Regula, G.; Meylan, M. Effects of management practices, animal transport and barn climate on animal health and antimicrobial use in Swiss veal calf operations. Prev. Vet. Med. 2019, 167, 146-157. [CrossRef] [PubMed]

22. Catry, B.; Dewulf, J.; Maes, D.; Pardon, B.; Callens, B.; Vanrobaeys, M.; Opsomer, G.; De Kruif, A.; Haesebrouck, F. Effect of antimicrobial consumption and production type on antibacterial resistance in the bovine respiratory and digestive tract. PLoS ONE 2016, 11, e0146488. [CrossRef] [PubMed]

23. Swissmedic Swiss Agency for Therpeutic Products. 2020. Available online: https://www.swissmedic.ch/swissmedic/en/home/ about-us/swissmedic--swiss-agency-for-therapeutic-products.html (accessed on 28 August 2020).

24. Mzyk, D.A.; Bublitz, C.M.; Martinez, M.N.; Davis, J.L.; Baynes, R.E.; Smith, G.W. Impact of bovine respiratory disease on the pharmacokinetics of danofloxacin and tulathromycin in different ages of calves. PLoS ONE 2019, 14, e0218864. [CrossRef] [PubMed]

25. Lin, Z.; Cuneo, M.; Rowe, J.D.; Li, M.; Tell, L.A.; Allison, S.; Carlson, J.; Riviere, J.E.; Gehring, R. Estimation of tulathromycin depletion in plasma and milk after subcutaneous injection in lactating goats using a nonlinear mixed-effects pharmacokinetic modeling approach. BMC Vet. Res. 2016, 12, 1-10. [CrossRef] [PubMed]

26. Mclaughlin, C.; Fielder, A.E.; Yancey, M.F.; Bowersock, T.L.; Robinson, J.A. Rapid and prolonged distribution of tulathromycin into lung homogenate and pulmonary epithelial lining fluid of Holstein calves following a single subcutaneous administration of $2.5 \mathrm{mg} / \mathrm{kg}$ body weight. Int. J. Appl. Res. Vet. Med. 2010, 8, 129-137.

27. Clothier, K.A.; Kinyon, J.M.; Griffith, R.W. Antimicrobial susceptibility patterns and sensitivity to tulathromycin in goat respiratory bacterial isolates. Vet. Microbiol. 2012, 156, 178-182. [CrossRef]

28. Landis, J.R.; Koch, G.G. The Measurement of Observer Agreement for Categorical Data. Biometrics 1977, 33, 159-174. Available online: http:/ / www.jstor.org/stable/2529310 (accessed on 2 May 2020). [CrossRef] [PubMed]

29. Greve, W.; Wentura, D. Wissenschaftliche Beobachtung: Eine Einführung; Psychologie Verlags Union: Weinheim, Germany, 1997; ISBN 3-621-27360-3.

30. Cohen, J. A Coefficient of Agreement for Nominal Scales. Educ. Psychol. Meas. 1960, 20, 37-46. [CrossRef]

31. Echtermann, T.; Müntener, C.; Torgerson, P.; Sidler, X.; Kümmerlen, D. Etablierung von definierten Tagesdosierungen und definierten Gesamtbehandlungsdosierungen zur Messung des Antibiotikaverbrauchs in der Schweizer Schweineproduktion. Schweiz. Arch Tierheilkd 2018, 160, 597-605. [CrossRef]

32. Echtermann, T.; Muentener, C.; Sidler, X.; Kümmerlen, D. Antimicrobial Drug Consumption on Swiss Pig Farms: A Comparison of Swiss and European Defined Daily and Course Doses in the Field. Front. Vet. Sci. 2019, 6. Available online: https: / / www.frontiersin.org/article/10.3389/fvets.2019.00240/full (accessed on 2 May 2020). [CrossRef] [PubMed]

33. Timmerman, T.; Dewulf, J.; Catry, B.; Feyen, B.; Opsomer, G.; de Kruif, A.; Maes, D. Quantification and evaluation of antimicrobial drug use in group treatments for fattening pigs in Belgium. Prev. Vet. Med. 2006, 74, 251-263. [CrossRef] [PubMed]

34. Schönecker, L.; Schnyder, P.; Overesch, G.; Schüpbach-Regula, G.; Meylan, M. Associations between antimicrobial treatment modalities and antimicrobial susceptibility in Pasteurellaceae and E. coli isolated from veal calves under field conditions. Vet. Microbiol. 2019, 236, 108363. [CrossRef] [PubMed] 